\title{
Sédentarisation des éleveurs transhumants dans le Nord du Cameroun : évolution des conflits ruraux et de leurs modes de résolutions
}

\author{
Natali Kossoumna Liba'a \\ Enseignant chercheur, Département de Géographie, Université de Maroua \\ B.P 55 Maroua, Tel : +237 778448 22, e-mail : kolibaa@yahoo.fr
}

\section{Résumé : Sédentarisation des éleveurs transhumants dans le Nord du Cameroun : évolution des conflits ruraux et de leurs modes de résolutions}

La fixation des éleveurs auprès des agriculteurs a fait apparaître de nouveaux conflits, mais aussi de nouvelles formes de médiation. Les acteurs impliqués dans ces conflits sont : les éleveurs, les agriculteurs, les chefs traditionnels et l'Etat. La proximité géographique est une source de multiplication des conflits mais concoure à leur atténuation car les éleveurs ne sont plus considérés comme des migrants perpétuels, prêts à causer des dégâts et à s'enfuir. Les règlements à l'amiable sont favorisés par le fait que chacun est assuré qu'il sera indemnisé, mais aussi grâce à la proximité géographique qui créée les relations de confiance, de tolérance, d'échange et de complémentarité. L'administration et son arsenal de mesures (lois et commissions) ainsi que les autorités traditionnelles sont entrain de disparaitre des modes de règlement à cause de leur inefficacité, leur lenteur, leur arbitraire et les coûts élevés des médiations.

Mots clés : Sédentarisation, conflits, eleveurs, agriculteurs, NordCameroun

\section{Abstract: Settlement of the transhumant stockbreeders in the North of Cameroon: evolution of the rural conflicts and their modes of resolutions}

The fixing of the breeders near the farmers revealed new conflicts, but also new forms of mediation. Actors implied in these conflicts are: breeders, farmers, traditional authorities and the State. The geographical proximity is a source of multiplication of the conflicts but contributes to their attenuation because the breeders are not regarded any more as perpetual migrants, ready to cause damage and to flee. The amicable settlements are supported by the fact that each one is assured that it will be compensated, but also stimulated by the geographical proximity related to the fixing of the breeders and the relations of confidence, tolerance, exchange and complementarities. The administration and its arsenal of measurements (laws and commissions) as well as the traditional authorities are spirit of disappearance modes of payment because of their inefficiency, their slowness, their arbitrary and the high costs of the mediations. The initiatives of regulation to friendly between actors thus much are.

Keywords: Settlement, conflicts, stockbreeders, farmers, North-

Cameroon 


\section{Introduction}

Les éleveurs ont communiqué avec les agriculteurs sédentaires depuis des millénaires, avec les pratiques établies de production commerciale et complémentaire comme le pâturage de bétail sur les champs des agriculteurs avant la saison de culture (Fratkin 1997). Pourtant, la croissance démographique et l'augmentation de la production marchande ont causé l'expansion d'agriculture sur les terrains de pâturage, les descentes massives des éleveurs transhumants des zones sèches vers celles plus humides ainsi que de nouvelles formes de mobilité des troupeaux pour s'adapter à la forte inégalité spatio-temporelle des ressources pastorales et hydriques (Sougnabe 2003). Ce qui a contribué à augmenter les tensions et les conflits entre les agriculteurs et les éleveurs dans beaucoup de parties du monde. Ces conflits ont non seulement un impact direct sur les vies et les moyens d'existence de ceux qui sont impliqués, mais ils désorganisent aussi et menacent la durabilité des systèmes de production agricole et pastorale (Moritz 2006). Comme dans toute l'Afrique Subsaharienne, les conflits sont fréquents entre les éleveurs et les agriculteurs dont les intérêts et les pratiques diffèrent fortement dans le bassin de la Bénoué dans le Nord du Cameroun. En effet, la concurrence entre l'élevage et la production agricole par la conversion de la terre de pâturage en terre cultivable d'une part, et l'expansion de l'agriculture irriguée le long des points d'eau, notamment la Bénoué, limitant l'accès à l'eau et au pâturage en saison sèche d'autre part, commencent à prendre une ampleur considérable. En cultivant les pistes de bétail et les zones réservées aux pâturages (enrichis par le fumier), les agriculteurs sédentaires limitent la mobilité des troupeaux des éleveurs causant des conflits en cas de piétinement et de destruction des cultures. Des expériences de régulation des conflits agropastoraux ont été effectuées par la préservation de parcours et par l'aménagement concerté du territoire avec l'appuie de commissions paritaires émanant de la société civile qui s'est positionnée en parallèle aux instances administratives (Teyssier et al. 2002). Cette commission paritaire avait l'avantage d'impliquer les différentes communautés et d'aboutir à une régulation consensuelle obtenue après débats. Elle avait pour inconvénient de manquer d'autorité pour faire respecter ses délibérations, et ses membres ne résistaient pas longtemps à l'usure du bénévolat. Quand le conflit était trop vif, la Commission éprouvait des difficultés à trouver des compromis. Des conventions locales légitimes devant les populations concernées ne sont donc pas forcement des dispositions légales devant les instances de l'Etat. La présence de représentants des services techniques lors du processus d'élaboration et de validation locale des conventions locales n'est pas suffisante pour leur conférer la sécurité juridique nécessaire. Elle ne représente qu'une caution morale vis-à-vis de l'initiative des populations, mais elle n'est pas égale à une légalisation quelconque. Les autorités traditionnelles quant à elles étaient très réticentes au début de l'opération (Graefen et al. 2005). Ces conventions restent donc fragiles et fortement dépendantes des structures des projets qui supportent souvent entière- 
ment le coût des interventions. Ces démarches qui viennent se greffer ou supplanter les instances existantes (autorités traditionnelles, administratives, judiciaires) jouent encore un rôle limité. Avec la proximité géographique au sens de distance - qu'ont aujourd'hui les éleveurs avec les agriculteurs, nous assistons à la consolidation des relations d'échanges et de complémentarité mais aussi à l'émergence de nouvelles formes de règlement des conflits que nous nous proposons d'analyser dans le cadre de cet article.

\section{Revue de la littérature et cadre théorique}

Les conflits auxquels nous nous intéressons se distinguent par leur caractère localisé (c'est-à-dire superposition territoriale d'intérêts contradictoires, rivalités entre espaces contigus ou proches), par la matérialité de leur support ou de leur objet d'intérêt, ainsi que par le fait qu'ils naissent à propos d'usages différenciés de l'espace (Torre et al. 2010). II s'agit ici des conflits ouverts dont l'existence est connue et reconnue par les acteurs sociaux. II s'agit par exemple d'une dispute entre deux individus ou d'une bagarre entre deux villages (Hagberg 200I). Ce sont donc des conflits à petite échelle facilement identifiable et entre relativement un petit nombre de personnes.

Dans les études sur les conflits agriculteurs-éleveurs, la littérature montre quelques problèmes méthodologiques relevés par Moritz (2006). II s'agit par exemple du fait que les chercheurs sont souvent affiliés avec une des parties impliquées dans le conflit posant le problème des données à collecter et leur interprétation. Un autre enjeu méthodologique, c'est qu'il est difficile d'avoir des données fiables sur l'évolution du nombre de conflits (Hussein et al. 2000) même si Moritz (2006) relève qu'il est facile de documenter les aspects plus qualitatifs de conflits agriculteurs-éleveurs. Enfin, de nombreuses analyses sont fondées sur de comptes rendu contradictoires, des informations incomplètes et des observations partielles.

On retrouve ensuite dans la littérature de nombreuses études de cas pour illustrer des modèles généraux et des évolutions de conflits agriculteurs-éleveurs. En général, les études empiriques plus détaillées des contextes écologiques de conflits sont nécessaires pour examiner le rôle de la dégradation de l'environnement sur l'évolution de la pression et de la compétition sur les ressources naturelles. En effet, depuis les années 70 , dans la zone soudanosahélienne, les tensions et conflits entre agriculteurs et éleveurs ont toujours existé pour des raisons variées : remontée du front pionnier agricole en période de pluviométrie normale, colonisation des abords des nappes d'eau par les agriculteurs, destruction des récoltes par les troupeaux nomades (Bernus 1974), mais également l'établissement des parcs naturels ou leur extension, le développement de l'irrigation (Moritz 2002). Avec la pression sur l'espace pastoral et la tendance à la sédentarisation, de nombreux éleveurs qui résident à la lisière d'un village pratiquent la transhumance du bétail bovin combinée avec l'agriculture céréalière de subsistance (Hagberg 200I). Par conséquent, la coexistence entre les groupes d'agriculteurs et les agropasteurs peuls est devenue de plus en plus 
problématique depuis les années 1980 . Les disputes, dues aux dégâts dans les champs, à l'accès aux points d'eau et aux passages des troupeaux, sont fréquentes, mais elles ne se limitent pas seulement à des causes matérielles. Hagberg (200I) relève que les identités ethniques et les droits des citoyens sont aussi des enjeux. Les disputes entre individus ou familles se sont transformées en conflit violent entre communautés villageoises et, pire, entre groupes ethniques. Dans ce cas, la distinction entre les " autochtones " et les " étrangers " s'accentue rapidement. D'autre part, les systèmes de mise en valeur de l'espace, tant pastoral qu'agricole, extensifs, les représentations sociales de l'espace antinomiques et surtout un interventionnisme de l'Etat en matière de gestion de l'espace sont évoqués comme causes des conflits entre les agriculteurs et les éleveurs dans les zones de savanes d'Afrique Centrale (Ankogui-Mpoko 2003). Ce qui montre que l'intégration territoriale entre élevage et agriculture n'est pas une réalité quand ces deux activités sont encore accomplies de façon dominante par deux communautés distinctes (Gauthier et al. 2005). Ainsi, les conflits d'usage de l'espace sont-ils caractérisés par une forte diversité d'expression en fonction des activités, des usages autour desquels ils prennent naissance, des territoires où ils se localisent, ainsi que des caractéristiques des acteurs qui les portent (Torre et al. 2010). D'autres auteurs analysent la place des autorités dans la gestion des ressources naturelles et des conflits. Moritz (2006) met ainsi en évidence un conflit entre les agriculteurs Tupuri et les pasteurs nomades de Fulbe dans l'Extrême-
Nord du Cameroun pour montrer comment les autorités traditionnelles et autorités d'Etat par leur 'politique de conflit permanent' continuent à activer des conflits entre pasteurs et agriculteurs.

Il se pose également dans la littérature la question de la classification des conflits agriculteurs-éleveurs : pourquoi et comment ces conflits se développent-ils ? Une étude diachronique de ces conflits montre la complexité des événements sociopolitiques qui évoluent dans le temps et dans l'espace. Ces interactions nous aident à comprendre pourquoi certains conflits s'intensifient et pourquoi d'autres s'estompent. Ce qui exigent une approche approfondie et au cas par cas pour mettre en œuvre les modes de leurs résolutions. De ce point de vue, il est important de lier l'évolution des conflits agriculteurséleveurs selon les générations. Deux publications majeures montrent qu'il ya une différence intergénérationnelle dans la perception des conflits. Elles montrent que les vieux et les jeunes ont une interprétation significativement différente des conflits. Dans le nord-ouest du Cameroun, Dafinger et Le Pélican (2002) ; Davis (1995) montrent que la jeune génération adopte une attitude plus offensive avec un détachement visà-vis du pulaaku' par rapport à une attitude plus passive des vieilles générations. Au centre du Ghana c'est principalement les jeunes agriculteurs qui poursuivent activement des stratégies plus conflictuelles et souvent violentes pour essayer de sécuriser l'accès au foncier face à la concurrence des bergers et des commerçants (Tonah 2006). 


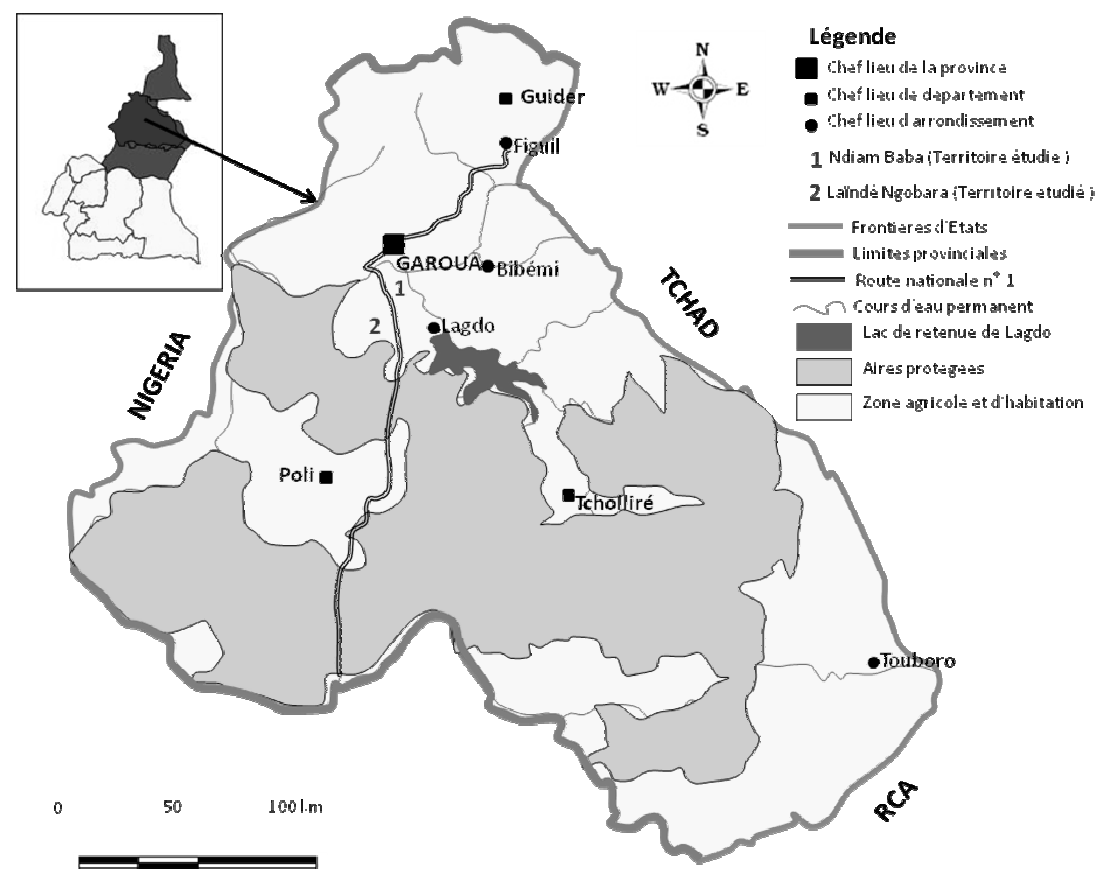

Figure 1. La zone d'étude

L'article proposé s'appuie sur le contexte d'évolution des conditions de vie et d'activité des éleveurs mbororo pour analyser l'évolution des situations conflictuelles avec les agriculteurs. En effet, avant la sédentarisation massive des éleveurs auprès des agriculteurs, les conflits étaient dans la plupart des cas gérés au niveau des chefs traditionnels, de la brigade de gendarmerie ou des autorités administratives et dans une moindre mesure par consensus entre les protagonistes. Il s'agit dans cet article de comprendre si la fixation des éleveurs et de leurs activités dans de petits territoires auprès des agriculteurs sédentaires peut être un facteur d'exacerbation ou de multiplication des conflits ou au contraire un facteur de leur atténuation. Par ailleurs, l'émergence de formes passive de régulation apparues au Nord Cameroun par rapport à ce qui se passait par le passé et par rapport à ce qui se passe en général dans d'autres pays sahéliens sont-elles durables ? Ces nouvelles formes de médiation doivent-elles être préférées ou encouragées?

\section{Données et méthode}

\section{La zone d'étude}

Notre zone d'étude comprend le bassin de la Bénoué et deux villages d'éleveurs Mbororo: Ndiam Baba créé en 198I et Laïndé Ngobara créé en 1995 (Kossoumna Liba'a 2008). La communauté Mbororo est une des composantes du grand groupe ethnique Peul d'Afrique subsaharienne. Ces deux villages constituent le territoire d'attache où se 
sont sédentarisés ces éleveurs. Ce choix permet de comparer deux dates de sédentarisation et deux situations, l'une proche d'une zone urbaine (Ndiam Baba situé à 12 kilomètres de Garoua) et l'autre au cœur d'une zone très agricole de production cotonnière (Laïndé Ngobara situé à environ 40 kilomètres au sud de Garoua).

Nous avons axé nos investigations et nos analyses sur deux villages mais nous ferrons appel parfois à nos connaissances du milieu et à quelques informations obtenues auprès des autorités administratives et coutumières, des responsables des services publics de la région pour étayer certains de nos propos.

\section{Les enquêtes auprès des exploitations}

Le niveau de l'exploitation est la concession qui constitue l'unité d'enquête.
Nous avons ainsi pris l'exploitation comme une unité de production familiale qui se résume à l'ensemble regroupant un homme marié (chef de ménage), son (ses) épouse(s), leurs enfants et d'éventuels dépendants directs, les parcelles en propriété, le cheptel animal et l'ensemble des activités extra-agricoles. A ce niveau, nous avons d'abord fait un recensement exhaustif de tous les chefs d'exploitation au nombre de 36 à Laïndé Ngobara et 28 à Ndiam Baba. Une typologie d'exploitations a été effectuée à l'aide de quelques variables discriminantes (âge moyen du chef d'exploitation, superficie moyenne en ha, superficie cultivée en ha par actif, nombre moyen de bovins, nombre de petits ruminants). Nous avons ainsi constitué 4 types d'exploitation (Tableau I).

Tableau I Ensemble des exploitations suivies

\begin{tabular}{l|c|c|c}
\hline \multicolumn{1}{|c|}{ Types d'éleveurs } & $\begin{array}{c}\text { Ndiam } \\
\text { Baba }\end{array}$ & $\begin{array}{c}\text { Laïndé } \\
\text { Ngobara }\end{array}$ & Total \\
\hline $\begin{array}{l}\text { Jeunes éleveurs en début de carrière avec peu de bovins cultivant } \\
\text { de petites surfaces (Type I) }\end{array}$ & 4 & 5 & 9 \\
$\begin{array}{l}\text { Eleveurs avec d'assez grand troupeau cultivant d'assez grandes } \\
\text { surfaces (Type 2) }\end{array}$ & 3 & 2 & 5 \\
$\begin{array}{l}\text { Eleveurs âgés avec peu de bovins confiés à leurs proches et cultivant } \\
\text { de petites surfaces (Type 3) }\end{array}$ & 4 & 5 & 9 \\
$\begin{array}{l}\text { Eleveurs avec de grand troupeau sans production végétale (Type 4) } \\
\text { Total échantillon }\end{array}$ & 2 & 2 & 4 \\
\hline Nombre total d'unités de production recensées & $\mathbf{2 8}$ & $\mathbf{3 6}$ & $\mathbf{6 4}$ \\
\hline
\end{tabular}

Les 3 premiers types correspondent grosso modo au cycle de vie d'un éleveur et ont réussi à combiner utilement les deux activités (agriculture et élevage). Du point de vue théorique, il est important de voir comment ces types d'éleveurs perçoivent les conflits liés aux dégâts champêtres et comment ils se représentent les relations avec les agriculteurs et les territoires d'activités qu'ils utilisent. En effet, les stéréotypes locaux selon lequel le peul est un " migrants perpétuel " est entrain de s'estomper du moment où les activités http://aps.journals.ac.za 
agricoles et pastorales - ainsi que les gens qui s'y engagent - s'entremêlent davantage aujourd'hui qu'auparavant sur le plan pratique (Habgerg 200 I). Frantz (1990) ; De Bruijn et Van Dijk (1995) proposent que la distinction entre agriculteurs et pasteurs ne soit qu'une question de grade; chaque groupe doit trouver un équilibre entre mobilité/ sédentarité et pastoralisme/agriculture.

Le type 4 quant à lui ne partage pas les mêmes représentations territoriales avec ceux qui pratiquent l'agriculture. II a une représentation linéaire du territoire à la recherche de points d'eau et de pâturages (Gauthier et al. 2005), n'a pas le même rapport avec les plantes cultivées et avec les agriculteurs. Ainsi, selon leur position, leurs intérêts et de leurs valeurs, tous les acteurs n'ont pas la même perception des conflits (Hagberg 200I).

\section{L'observation passive et suivi des animaux en pâturage}

Dans le cadre de notre recherche, nous avons procédé à une observation passive avec des visites fréquentes et des séjours répétés dans les deux territoires (Tableau II).

Notre démarche a consisté en l'accompagnement de chaque type d'éleveurs au moins une fois par semaine pendant un an dans leurs trajets quotidiens et dans l'accomplissement de leur travail afin d'observer leurs faits, gestes et comportements, mais aussi afin de nous imprégner des réalités des villages et des vécus quotidiens des éleveurs, de recueillir de manière spontanée les différentes informations et de comprendre certaines subtilités propres à chaque territoire.

Tableau II Nombre de suivis effectués par village et par type d'éleveurs

\begin{tabular}{ccc}
\multicolumn{3}{c}{ en 2006 } \\
\hline Ndiam Baba & Laïndé Ngobara \\
\hline Type I & I I & 9 \\
Type 2 & 8 & 11 \\
Type 3 & 9 & 10 \\
Type 4 & 11 & 9 \\
\hline Total & 39 & 39 \\
\hline
\end{tabular}

Source : Enquêtes, Kossoumna Liba’a (2008)

Des rencontres informelles ont eu lieu également au moins une fois par semaine avec une trentaine d'habitants de six villages voisins aux éleveurs mbororo. Dans un souci d'observation, nous avons assisté à 26 séances de résolution des conflits dans les deux villages d'éleveurs et les villages d'agriculteurs voisins.

L'étude du déplacement des troupeaux au sein des différents territoires nous donne de précieuses informations sur les conflits rencontrés sur les parcours. Ces suivis nous ont permis d'obtenir des informations très précises sur les différentes formes de compétition et de conflits entre les acteurs, leurs fréquences, leurs coûts et les formes de médiations privilégiées par les protagonistes en présence. Les donurnals.ac.za 
nées ont été dépouillées manuellement. Selon les thèmes abordés, les réponses ont été regroupées par types d'éleveur et par modalités : types de conflits rencontrés, fréquences des conflits, modes de résolution, instance de résolution, coûts de règlement.

\section{Résultats}

Les acteurs concernés par les conflits

La notion d'acteurs ici n'est pas réductrice aux seuls éleveurs et agriculteurs mis en exergue dans ce genre d'analyse. Elle concerne tous ceux qui sont impliqués dans les causes des conflits et les modes de résolution. II s'agit Premièrement, les éleveurs mbororo à Ndiam Baba et à Laïndé Ngobara dont les mobilités et la possession ou la garde encore importante de bétail les placent au centre des conflits sur l'accès et la gestion des ressources naturelles et des territoires. Deuxièmement, les agroéleveurs des villages voisins de Ndiam Baba et de Laïndé Ngobara, ainsi que ceux qui se trouvent le long des espaces de pâturage utilisés par les éleveurs pour la transhumance, sont constitués exclusivement des personnes sédentaires. Leurs activités sont en grande partie bâties sur la culture vivrière et/ou de rente avec un élevage limité à quelques bœufs de trait et de petits ruminants. Troisièmement, la chefferie traditionnelle comprend le laamii'do ${ }^{2}$ et ceux qui la représentent et décident en son nom comme le sarkin saanou ${ }^{3}$ ou encore à l'échelle du village à savoir les lawan ou ar'do ${ }^{4}$ et les jawro ${ }^{5}$. Dans toutes les grandes chefferies du NordCameroun en effet, l'accès à la terre est contrôlé par l'autorité traditionnelle qui n'accorde aux exploitants que des droits précaires et prélève des redevances sur les récoltes. C'est elle également qui, jusqu'alors, est chargée des règlements de litiges. Quatrièmement, nous avons lEtat et ses représentants qui regroupent ici les administrations déconcentrées et leurs services techniques. II s'agit au niveau de l'arrondissement de Tchéboa du sous-préfet, des chefs de services techniques, en particulier du délégué de l'agriculture et du développement rural, du délégué de l'élevage, des pêches et des industries animales, du chef de service départemental des domaines, celui du Cadastre, celui de l'urbanisme et de l'habitat. Enfin, nous avons des collectivités locales dont les représentants sont élus (le maire et son conseil municipal). Ces deux derniers acteurs font partie de la commission de règlement des litiges agro-pastoraux.

\section{Les différents types de conflits fréquemment rencontrés}

Les conflits d'usage de l'espace sont caractérisés par une forte diversité d'expression en fonction des activités, des usages autour desquels ils prennent naissance, des territoires où ils se localisent, ainsi que des caractéristiques des acteurs qui les portent (Torre et al. 2010). Dans le cadre de cette recherche, en dehors des conflits classiques entre éleveurs et agriculteurs, il y a également les conflits entre les autorités traditionnelles et les éleveurs.

Le conflit entre les agriculteurs et les éleveurs La fixation des éleveurs avec leurs animaux auprès des agriculteurs a engendré la multiplication et la diversification des conflits sur les ressources naturelles et les territoires (Figure 2). 


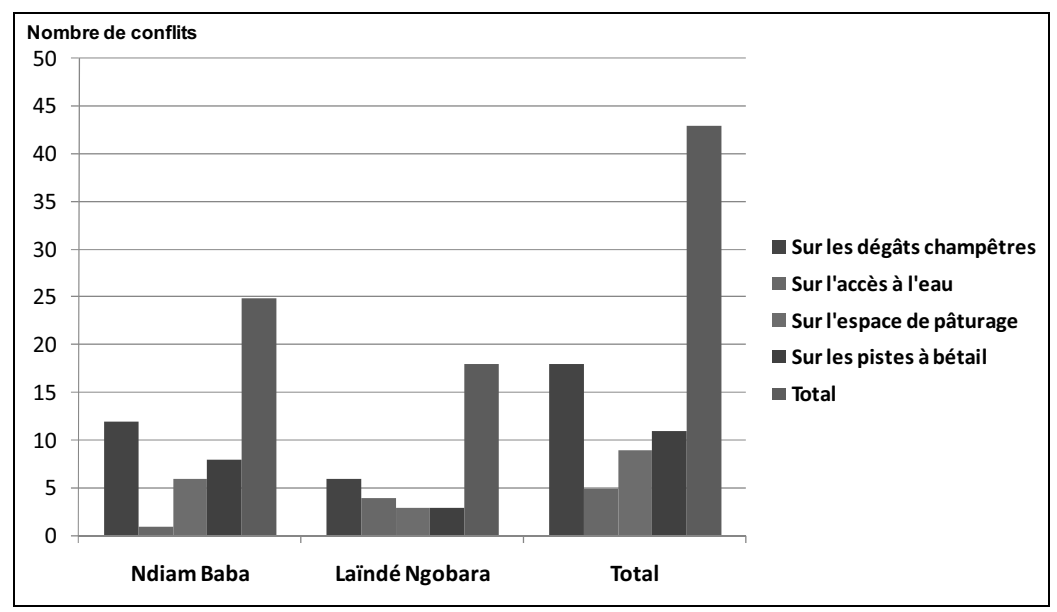

Figure 2. Nombre de types de conflits total recensé à Ndiam Baba et Laïndé Ngobara en 2006

Source : Enquêtes, Kossoumna Liba'a (2008)

Alors qu'auparavant les conflits étaient concentrés pendant la période de récolte à cause des dégâts sur les cultures, nous assistons non seulement à leur étalement continu au cours de l'année mais aussi à l'augmentation de leur fréquence. De même, les éleveurs ne sont plus les seuls à provoquer les conflits. Avec la délimitation et le bornage des espaces de pâturage et des pistes à bétail, les agriculteurs qui y cultivent sont également à la source de nombreux conflits.

Quatre principaux motifs de conflits entre les agriculteurs et les éleveurs sur les ressources naturelles et les territoires ont été évoqués (Figure 2) : les dégâts sur les cultures, l'accès à l'eau, les conflits relatifs à la mise en culture par des agriculteurs des parcours naturels et des pistes à bétail où les agriculteurs ont tendance à mettre des champs. Ainsi, la compétition entre les éleveurs et les agriculteurs, au-delà de l'accès aux ressources, peuvent aussi être vues comme une compétition entre deux différents systèmes de production ou d'utilisation de l'espace : agriculture et élevage (Moritz 2006). En fait, la compétition entre les systèmes de production a été considérée inhérente à la coexistence des deux systèmes de production (van den Brink et al. 1995) et la cause ultime de conflits agriculteurs-éleveurs (Hussein et al. 2000).

Les dégâts champêtres sont deux fois plus fréquents à Ndiam Baba qu'à Laïndé Ngobara. En effet, contrairement à Laïndé Ngobara assez isolé des champs, Ndiam Baba est entouré de manière très rapprochée des villages d'agriculteurs et de leurs champs. Ce qui les expose plus à des dégâts sur les cultures.

Les conflits liés à l'accès à l'eau sont plus importants à Laïndé Ngobara qu'à Ndiam Baba. Ce dernier territoire est situé non loin de la Bénoué et les éleveurs transhument depuis longtemps au 
bord de ce fleuve. L'espace sur lequel ils parquent leurs animaux au bord de la Bénoué est reconnu par les agriculteurs qui n'y pratiquent pas l'agriculture de décrue. Ce n'est pas le cas pour les éleveurs de Laïndé Ngobara dont la transhumance sur ces lieux est plus récente et pour lesquels les relations avec les agriculteurs ne sont pas encore bien établies.

\section{Les dégâts champêtres}

Les conflits liés aux dégâts champêtres en nette progression ces dernières années sont au centre des préoccupations tant des agriculteurs que des éleveurs qui se rejettent les responsabilités. Ils représentent près de la moitié des cas évoqués dans les deux territoires. Pour les agriculteurs cette situation résulte du refus délibéré des éleveurs de respecter les usages établis en la matière. Les éleveurs sont accusés de rester plus longtemps avec leurs animaux dans leurs territoires d'attache (fin juillet - début août) alors que les cultures des agriculteurs des villages voisins sont déjà bien développées. Les éleveurs sont en effet censés emmener le gros de leur troupeau en transhumance.

D'autre part, les agriculteurs disent que les éleveurs ne respectent plus « la date de libération des champs" pour entreprendre le retour de la transhumance. Les agriculteurs accusent les éleveurs mbororo de laisser sciemment leurs animaux dévorer la partie non récoltée d'un champ. C'est la raison pour laquelle on leur interdit systématiquement la vaine pâture quand une parcelle n'est pas totalement récoltée.

Les éleveurs quant à eux estiment que la question des dégâts champêtres, au delà de ces aspects, est aussi liée à la restriction d'un certain nombre de droits à eux implicitement reconnus. II s'agit notamment les couloirs de passage et aires de repos et de parcage qui ont été systématiquement colonisés par les champs rendant ainsi périlleux le déplacement des troupeaux et la limitation des possibilités des animaux pour trouver des aires de repos. Ils se plaignent aussi de certains actes que posent, selon eux, délibérément les agriculteurs pour les amener à commettre les dégâts. II s'agit des " champs pièges " ou " champs de provocation " autour des rares mares dans les flancs des montagnes, zone par excellence réservée à l'élevage où les animaux se réfugient pendant la saison pluvieuse pour rechercher le pâturage. Voyant leurs pâturages se réduire peu à peu devant l'avancée des emblavures de coton et de maïs, les éleveurs mbororo réagissent par des mouvements de provocation en faisant passer délibérément leurs troupeaux sur les champs car ils arrivent difficilement à se discipliner, à tenir compte des blocs de culture et à ne pas faire de " hors-piste " à bétail.

Les problèmes des champs de " coton récoltés tardivement " ont été également signalés par les éleveurs. En effet, les pistes sont fermées plus longtemps que prévu, bloquées par des champs non récoltés, jusqu'en décembre - janvier. Les agriculteurs utilisent cette méthode pour pousser les éleveurs à partir ou à ne plus utiliser certains espaces de pâturage.

La mise en culture progressive des couloirs à bétail et des parcours

Au cours de chaque saison des pluies, des conflits opposent les agriculteurs et les éleveurs pour le respect de l'espace 
délimité et réservé au pâturage. Ces conflits sont provoqués par le fait que les agriculteurs n'ont pas cessé d'y cultiver. Or, les espaces réservés aux bétails appartiennent en principe autant aux éleveurs qu'aux agriculteurs. Mais, les éleveurs sont plus engagés dans la préservation de ces espaces parce qu'ils ont plus d'animaux. Se sachant dans leurs droits, ils ne se privent pas d'y pâturer. Malgré les conventions mises en place, rien n'est fait à l'encontre des agriculteurs qui cultivent les parcours et les pistes. Lorsque des dégâts sont perpétrés dans les champs, les agriculteurs portent plainte surtout auprès des autorités traditionnelles qui ne se privent pas de légiférer afin de bénéficier des avantages liés aux règlements des litiges ${ }^{6}$.

Les couloirs à bétail (burti) sont également au centre des conflits agropastoraux notamment lors de transhumances pour lesquelles les éleveurs ont besoin de passages pour pouvoir migrer avec les troupeaux à la recherche de pâturages ou pour accéder aux points d'eau. Ceux-ci sont de moins en moins accessibles et ouverts. Entre octobre et janvier, en effet, les champs de maïs et coton qui ne sont pas encore récoltés compliquent les départs en transhumance, tandis qu'en juin, leur retour est empêché par les champs nouvellement semés.

La disparition de certains couloirs a amené très souvent les éleveurs à emprunter les routes goudronnées et les pistes empruntées par les automobiles exposant ainsi les animaux et les autres usagers de la route aux accidents et donc à une autre amende liée à la divagation des animaux. En plus, les risques de dégâts champêtres sont toujo- urs réels car les champs aux abords des routes ne sont pas clôturés et les paysans ne se privent pas des terres profondément remuées et fertiles des environs de ces routes.

Inversement des automobilistes ont transformé certains couloirs de passage en piste automobile pour fuir le mauvais état des pistes latéritiques, pour avoir des raccourcis ou encore pour fuir la gendarmerie.

\section{L'obstruction de l'accès à l'eau}

Des conflits surviennent également le long des cours d'eau où les animaux s'abreuvent lorsqu'ils sont en transhumance ou hors de leurs territoires d'attache. En effet, le plus souvent, les cultures maraîchères cultivées au bord de l'eau ne sont pas clôturées. Dans ces zones de passage incontournables pour abreuver les animaux, les éleveurs n'arrivent pas toujours à contenir leurs animaux.

L'accès aux autres points d'eau (forage, mare, puits) constitue l'autre aspect majeur du conflit entre éleveurs mbororo et agriculteurs. En effet, la mauvaise répartition des puits pastoraux, le non fonctionnement de la plupart, l'assèchement précoce des mares font de l'accès à l'eau un enjeu majeur dans la gestion des conflits. La non disponibilité de l'eau dans des zones à forte production de biomasse herbacée amène les éleveurs à se rabattre dans ces zones agricoles plus nanties. Or, en ces lieux, sous la pression démographique et la rareté des ressources naturelles, s'est développée une appropriation privative de ces points d'eau et des bas-fonds environnant occupés par les champs ${ }^{7}$. De plus, les cultures de décrues bloquent l'accès à la Bénoué jusqu'en mars/avril pour les 
éleveurs de Laïndé Ngobara.

De même que les causes des conflits diffèrent d'un territoire à l'autre, elles le sont également d'un type d'éleveur à un autre (Figure 3).

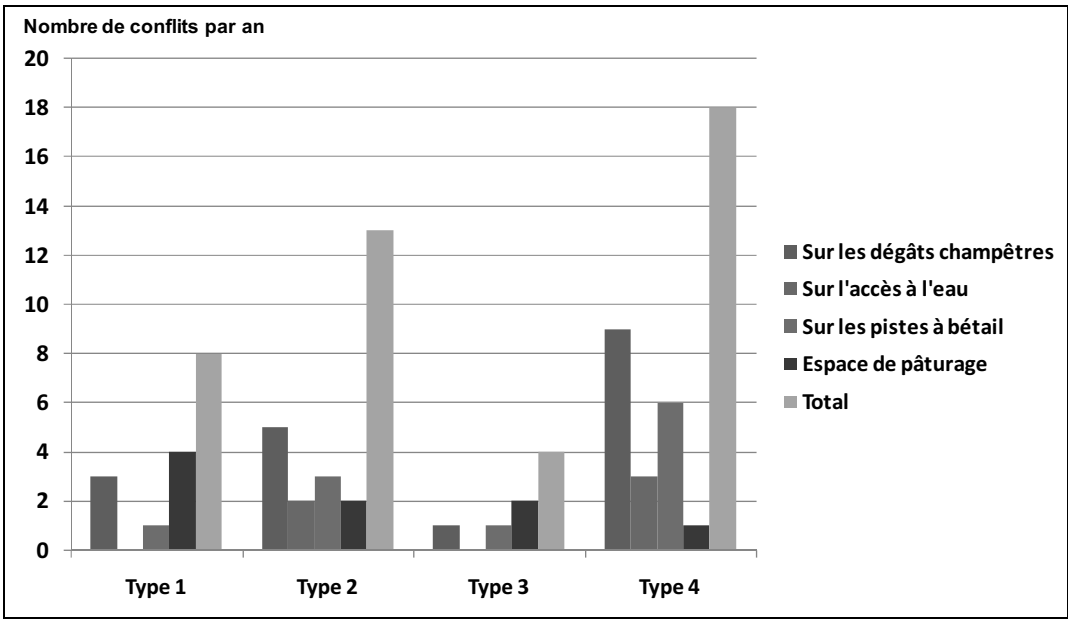

Figure 3. Moyenne des types de conflits par type d'exploitation à Ndiam Baba et Laïndé Ngobara

Source : Enquêtes, Kossoumna Liba'a (2008)

Pour les types I et 3, les conflits sont limités probablement parce que le nombre moins important d'animaux leur permet de bien les surveiller. A cela il faut ajouter pour le type I le fait que ces éleveurs gardent eux-mêmes leurs animaux. Pour le Type 2 et le Type 4, le nombre élevé de têtes de bétail est proportionnel au nombre de conflits enregistrés au cours de l'année. Pour limiter les conflits, ces types scindent le bétail en plusieurs troupeaux chacun sous la garde d'un berger. Mais si les conflits persistent, les bergers en sont la cause car selon les éleveurs, ils ne sont pas toujours vigilants parce que les dommages dans les champs ne leur sont pas imputés. Ce sont toujours les propriétaires qui paient. Moritz (2006) fait ainsi une distinction entre les bergers avec les bâtons et les bergers avec les familles (gaynaako bee sawru e gaynaako bee saare). Les bergers avec les bâtons (waynaaBe bee cabbi) ont peu d'intérêt pour leurs troupeaux et vont de job en job (et de patron en patron). Les bergers avec les familles (waynaaBe bee caalaaje), d'autre part, ont plus de responsabilités et prendront mieux bon soin des troupeaux que de jeunes bergers qui ne possèdent rien d'autre que leur bâton et leurs vêtements sur eux.

De plus, à cause de leur grande mobilité, les éleveurs de Type 4 sont beaucoup plus exposés aux conflits notamment ceux liés aux dégâts champêtres qui représentent la moitié des cas évoqués. Ces éleveurs transhumants sont également les plus confrontés aux conflits sur les pistes à bétail.

Conflits entre les autorités traditionnelles et 
les éleveurs

Sans prendre un caractère ouvert, les conflits entre les différents niveaux des autorités traditionnelles et les éleveurs sont fréquents. Le ressentiment et la rancœur sont grands dans les communautés d'éleveurs par rapport à l'application de l'échelle des peines et sanctions en cas de dégâts champêtres. Dans beaucoup de cas, c'est la victime ellemême qui se voit accorder la latitude de fixer unilatéralement le montant des dommages et intérêts à lui dus. II n'y a pas de tarification connue et unanimement acceptée sur les frais de prise en charge des dégâts. Les montants à payer sont fixés à la tête du client. Les éleveurs estiment que les sanctions appliquées aux agro-éleveurs sont moins importantes car à l'inverse les sévices et les mauvais traitements infligés à leur bétail sont rarement indemnisés puisque le rapport de force n'est pas en leur faveur. Les nombreux cas portés à notre connaissance montrent qu'en cas de contestation de l'estimation des dégâts c'est l'agriculteur qui a le dernier mot. Ce qui a fait dire au Délégué d'arrondissement de l'élevage, des pêches et des industries animales de Tchéboa que "l'agriculteur n'a jamais tort. Ils sont là pour provoquer et s'ils provoquent, c'est l'éleveur qui paie "8. Ainsi, chez les éleveurs prévaut le sentiment qu'il y a une complicité agissante entre les chefs traditionnels et les agriculteurs, vivants souvent depuis très longtemps ensemble, contre les éleveurs mbororo encore instables dans la région. Leur errance et leur analphabétisme les rendent vulnérables comme l'exprime Ndoudi Oumarou : “ Quel que soit l'endroit où nous nous trouvons, aucune considération ne nous est due, à nous les Mbororo. Comment l'expliquer? Nous sommes des gens sans village et sans terre, des illettrés, peu instruits de notre religion, ne sachant rien des choses du monde [...]. Tel est notre sort, à nous gens de la brousse, nomades sans instruction, tout juste bons à être exploités en tous lieux et par tous ! » (Bocquéné 1986).

Les éleveurs reprochent également aux chefs traditionnels de ne pas toujours attirer l'attention de leurs administrés par rapport à des acquis de longue date notamment sur les couloirs de passage, les aires de pâturage colonisés par les agriculteurs qui n'hésitent pas à faire des " champs pièges " autour des mares et les cours d'eau traditionnellement réservés à l'élevage. C'est ainsi que dans l'espace de pâturage borné de Ndiam Baba, des agriculteurs possédant des parcelles ont déclaré qu'après la délimitation de cet espace, le laamii'do leur a demandé de reprendre l'exploitation de leurs parcelles au grand dam des éleveurs qui s'apprêtaient à cotiser de l'argent pour le sarkin saanou afin qu'il expulse ces agriculteurs.

\section{Mécanismes de règlement des conflits fréquemment utilisés}

Si tous les conflits n'éclatent pas, il faut souligner l'importance de tous les processus peu ou pas visibles par lesquels des acteurs en concurrence élaborent des compromis, renoncent à l'escalade d'un conflit possible et arrivent à cohabiter sur le mode de la tension et/ou la négociation, plutôt que celui de l'affrontement violent. Trois modes de règlement des conflits sont évoqués (Figure 4 ) : le règlement à l'amiable, le règlement auprès du jawro et le règlement 
auprès du laamii'do.

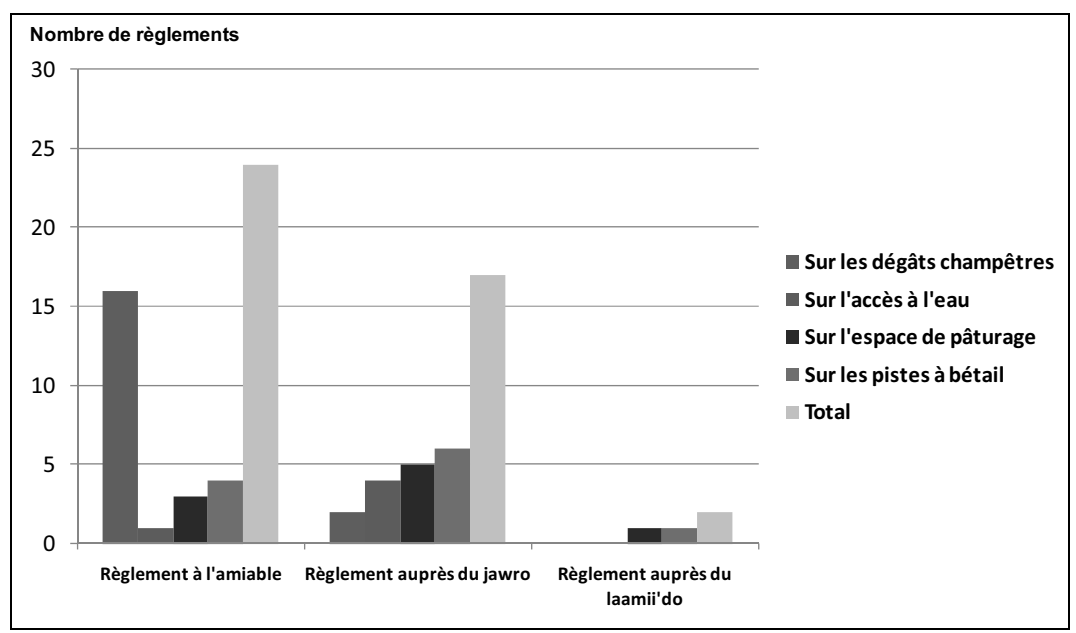

Figure 4. Modes de règlement des conflits par type de conflits rencontrés à Ndiam Baba et Laïndé Ngobara

Source : Enquêtes, Kossoumna Liba'a (2008)

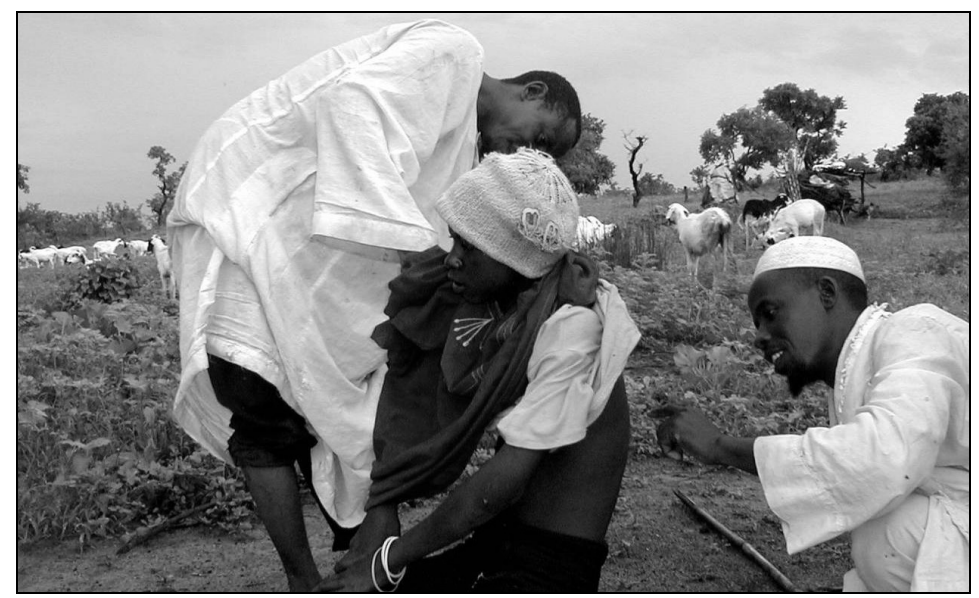

Photo 51. Les éleveurs constatent des blessures infligées à un jeune berger Mbororo par des agriculteurs à Lä̈ndé Ngobara

Cliché : Kossoumna Liba'a (2006)

La préférence aux règlements à l'amiable Plus de la moitié des conflits est réglée à l'amiable. En effet, les conflits entre les agriculteurs et les éleveurs ne sont plus sanglants. Bien que des sévices corporels soient encore exercés sur les bergers (Figure 5), nous n'assistons plus à mort d'homme comme ce fut le cas 
dans le passé.

La proximité géographique entre les deux protagonistes est sans doute un facteur déterminant dans la recherche et l'acceptation de négociation à l'amiable. En effet, plus les acteurs sont proches les uns des autres, mieux ils se connaissent et plus il est facile d'établir des responsabilités et d'engager des pourparlers en cas de conflits. Les éleveurs comme les agriculteurs affirment que les relations de bon voisinage qu'ils s'efforcent d'entretenir les poussent à régler l'affaire à l'amiable sans que l'administration publique ou les autorités traditionnelles soient impliquées ou, parfois même, informées. En dehors de l'activité pastorale et agricole, les éleveurs et les agriculteurs ont en effet des relations étroites au quotidien. Ils se côtoient tous les jours le long des routes autour d'un verre de thé ou de la viande grillée (soya), sur la place du marché, devant le moulin... Ce sont également les jeunes des villages voisins qui transportent les éleveurs mbororo pour les ramener dans leurs villages... Certains éleveurs aisés ont confié des motos-taxis aux jeunes agriculteurs des villages voisins. La fixation des éleveurs auprès des agriculteurs a également permis la consolidation des relations d'échanges et de complémentarité. En effet, les échanges étaient autrefois limités au troc entre produits d'élevage et produits vivriers, ou à la contrepartie entre fumure organique des champs et consommation fourragère de la vaine pâture. Ils se diversifient désormais et au troc, ont succédé les échanges commerciaux de produits alimentaires (mil, maïs, viande, lait...) et autres services (travaux agricole, confiage de bétail, construction de maison...). L'insécurité ambiante incite aussi les éleveurs à utiliser les bergers salariés issus des villages d'agriculteurs voisins. Ces derniers ont également accueillis les éleveurs mbororo fuyant les assauts répétés des preneurs d'otages. Tous ces facteurs contribuent à alléger les tensions qui surviennent et poussent chacun à accepter des règlements à l'amiable.

Règlement auprès du chef du village en cas de dégât important

Plus du tiers des conflits a nécessité l'intervention du chef du village (jawro) de la victime ou des deux villages. Ces conflits sont liés pour la plupart aux dégâts champêtres causés sur les pistes à bétail, au bord d'un point d'eau ou dans l'espace de pâturage et rarement sur les parcelles autour des villages. En fait, l'intervention du jawro survient lorsque les dégâts sont plus importants. Dans de tels cas, le constat est fait sur la parcelle le jour même où les dégâts se produisent ou le lendemain. Ceci pour ne pas amener les éleveurs à contester leur faute car d'autres troupeaux ont pu avoir le temps de pénétrer sur la parcelle. Le chef de village ou son représentant se rend dans le champ pour évaluer les dommages. Les rangées de cultures endommagées sont comptées. Les deux protagonistes s'entendent sur l'ampleur du dommage et la somme forfaitaire à verser sous la médiation du chef du village ou de son représentant. L'argent est remit au jawro qui le transmet à la victime. Contrairement à la norme auprès du laamii'do ou du sarkin saanou, le jawro n'est pas systématiquement rémunéré pour sa médiation. Cependant, la personne dédommagée peut de manière volontaire offrir au jawro une petite par- 
tie de la somme qui lui a été versée (environ les $\mathrm{I} / 10^{\mathrm{e}}$ ).

Les autorités traditionnelles et administratives de moins en moins sollicitées

Si un conflit n'est pas réglé à l'amiable et fait intervenir les autorités, ni l'agriculteur ni l'éleveur n'en sortent gagnants. Les éleveurs et les agriculteurs semblent avoir compris que pour l'intérêt de tous, la justice interne est préférable. Au cours de l'année 2006, seulement 2 conflits sur 42 enregistrés ont été réglé auprès du laamii'do ou de son représentant (sarkin saanou). Ce sont des conflits liés aux dégâts champêtres causés sur les pistes à bétail et les espaces de pâturage. La raison pour laquelle l'intervention de ces instances est de moins en moins sollicitée est qu'elle est rémunérée ${ }^{9}$ et nécessite souvent de nombreux déplacements coûteux. La résolution des conflits opérée par les chefs traditionnels en charge de ces litiges aboutit généralement à un équilibre précaire pouvant être ultérieurement remis en cause par les plaignants et donnant l'impression d'un choix délibéré pour des situations inachevées plutôt que des décisions bien tranchées. Ces chefs traditionnels puisent dans leur arsenal juridique composite, des arguments qu'ils opposent différemment aux acteurs selon leur statut sociopolitique (Gonné et al. 2010).

De même, aucun conflit n'a été porté auprès des instances officielles de règlements des litiges agro-pastoraux institutionnalisés au niveau de chaque arrondissement par le Décret $n^{\circ} 78 / 263$ du 03 septembre 1978. Cette commission regroupe des représentants de tous les acteurs (administrations, laamii'do, Ministère de l'Elevage, des pêches et des Industries Animales (MINEPIA) Ministère de l'Agriculture et du Développement Rural (MINADER), un représentant du Cadastre, agriculteurs, éleveurs,...) et est présidée par le sous-préfet. Ce décret fixe également le fonctionnement de la commission consultative sous-préfectorale qui devait normalement être dotée de moyens de fonctionnement (au moins pouvoir se déplacer sur le terrain). Ce qui n'est pas le cas. Aussi, ces commissions ne se réunissent pas sauf si les plaignants les payent. Les sous-préfets sont les " chefs de terre ", avec un statut de "diplomates pompiers " n'intervenant qu'en cas de conflits patents et risquant de créer des conflits sociaux violents pouvant mettre en péril l'ordre public dont il est garant. Ces commissions ne sont pas toujours acceptées par tous et n'empêchent pas les pratiques (ou les tentatives, plus ou moins fréquentes) de détournement ou de contournement. Les instances étatiques n'exercent de ce fait aucun contrôle. Les textes sont contournés. Le gouvernement ne diffuse ni n'applique les lois. Pourtant, l'Etat est un mari polygame qui doit considérer ses épouses (agriculteurs et éleveurs) avec égalité (Ouattara 1997). Ainsi, malgré la multiplication et l'augmentation de la fréquence des conflits, nous assistons à l'évolution des modes de règlements privilégiant la proximité et l'entente.

\section{Discussion}

Les conflits entre les différents acteurs émanent du fait qu'ils n'ont pas un sentiment partagé du sens à donner à l'intérêt collectif autour des espaces de pâturage, même délimités, qu'ils exploitent ensemble et des pistes à 
bétail. C'est pour cela qu'aucun consensus n'est à ce jour trouvé autour du respect de ces espaces, donnant lieu à de nombreux conflits. Les réglementations existantes ne sont pas respectées par les agriculteurs, de même qu'on assiste au laxisme des autorités tant traditionnelles qu'administratives. Face à tous ces acteurs, les éleveurs sont de véritables souffre-douleurs sans pouvoirs et sans soutiens.

\section{Conclusion}

La proximité géographique est une source de multiplication des conflits mais concourt à leur atténuation. Les éleveurs ne sont plus considérés comme des migrants perpétuels, prêts à causer des dégâts et à s'enfuir. Les règlements à l'amiable ont été également favorisés par le fait que chacun est assuré qu'il sera indemnisé. La mobilité constitue donc une source importante d'exposition à des conflits et à leur exacerbation.

L'administration et son arsenal de mesures (lois et commissions) sont entrain de disparaitre de ces modes de règlement. Les régulations auprès des instances étatiques et des autorités traditionnelles sont en effet inefficaces, lentes, arbitraires et coûteuses. Les initiatives de régulation à l'amiable entre acteurs sont donc beaucoup plus stimulées par la proximité géographique liée à la fixation des éleveurs et à la diversification des relations d'échanges et de complémentarité. Ce qui a favorisé l'établissement de relations sociales et économique entre les communautés d'éleveurs, les agriculteurs et les citadins. Les relations de confiance, de tolérance prennent ainsi le pas sur les tensions perpétuelles. Un autre enjeu du règlement à l'amiable est le gain financier et temporel que cela engendre. Les deux parties ayant compris que cela coutait cher de se plaindre auprès des autorités traditionnelles et administratives. Les perspectives, les intérêts et les valeurs à long terme qui entrent aujourd'hui en jeu sont telles que les conflits et les coopérations sont impossibles à éviter entre les agriculteurs et éleveurs du fait de la sédentarisation de ces derniers et leur implication dans l'agriculture. Comme le disait Seydou Badian (200I) " la langue et les dents, appelées à passer ensemble toute leur vie se querellent $»$. Les modes de règlement à l'amiable entre les deux parties est donc à encourager. Leur efficacité et durabilité dépendent de la volonté de chaque partie à adopter une attitude conciliante, de la complexification des liens d'interdépendance et de complémentarité entre eux. II reste à formaliser les choix des personnes susceptibles d'intervenir dans le règlement de ces conflits, les règles d'appréciation des dégâts causés et les modalités de leurs réparations.

\section{Notes}

I Pulaaku, défini par semteende, munyal et hakkiilo est une valeur identitaire des fulbe. Autrement dit, l'homme capable de régler ses actes avec retenue, réserve, pudeur, dans le sens de l'honneur et qui peut se sentir peiné, gêné, diminué en cas de transgression de cette ligne de conduite.

${ }^{2}$ Chef traditionnel de premier degré

3 Responsable des troupeaux du laamii'do et le représentant du laamii'do auprès des éleveurs

${ }^{4}$ Chef traditionnel de deuxième degré

5 Chef traditionnel de troisième degré 
6 En 2006, un éleveur de Ndiam Baba dont les animaux ont détruit les cultures installées sur la zone de pâturage a refusé de payer les amendes à lui infligées par l'ar'do de Babla. Se sachant dans son droit, il a porté l'affaire à la brigade de Tchéboa qui n'a pas donné suite à l'affaire. Sans se lasser, il a porté l'affaire auprès du tribunal de premières instances de Garoua où il a eu gain de cause. Ces cas de persévérance sont tout de même assez rares.

7 On le voit dans l'espace de pâturage de Kalgué et à Naddere où les éleveurs passent une bonne partie de leur période de transhumance.

${ }^{8}$ Communication personnelle le 26 juillet 2006

9 Le plaignant (agriculteur) verse 2000 Fcfa à la faada pour être introduit auprès du laamii'do, puis 5000 Fcfa pour que la commission lamidale se déplace sur le lieu du sinistre. Après le règlement du litige, s'il obtient gain de cause et s'il est dédommagé, il verse le $1 / 4$ de la somme perçue au laamii'do. L'éleveur quant à lui, pour ne pas être fortement amendé, verse de l'argent aux membres de la commission lamidale de règlement des litiges agro-pastoraux. Selon l'ampleur des dégâts, cette somme varie entre 20000 et 50 000 Fcfa.

\section{Bibliographie}

Ankogui-Mpoko, G-F. 2003. "Pratiques spatiales et conflits en zones de faible densité de population : Le cas du nord-est de Bambari en RCA" pp. 126-152. In Dugué P., Jouve Ph., (éds.), Organisation spatiale et gestion des ressources et des territoires ruraux. Organisation spatiale et gestion des ressources et des territoires ruraux. Actes du colloque, 25-27 février 2003, UMR SAGERT, CNEARC, Montpellier, France.

Bernus, E. 1974. "L'évolution récente des relations entre éleveurs et agriculteurs en Afrique tropicale : l'exemple du Sahel Nigérien". Cahiers ORSTOM, série Sciences Humaines, II(2): I37-I43.

Bocquene, H. 1986. Moi, un Mbororo : Ndoudi Oumarou, Peul nomade du Cameroun. Karthala, Paris. 387 p.

Dafinger, A et Pelican, M. 2002. Land rights and the politics of integration: pastoralists' strategies in a comparative view. Halle, Saale: Max Planck Institute for Social Anthropology. $210 \mathrm{p}$.

Davis, L. 1995. "Opening political space in Cameroon: the ambiguous response of the Mbororo". Review of African Political Economy, 22(64):2I 3228.

De Bruijn, M. et Van Dijk, H. 1995. Arid Ways: Cultural Understandings of Insecurity in Fulbe Society, Central Mali. Amsterdam, Thela Publishers. $167 \mathrm{p}$.

Frantz, C. 1990. "West African Pastoralism : Transformation and Resilience", pp. 293-337. In Salzman C et Galaty, J. G, eds, Nomads in a Changing World, Napoli, Dipartimento di studi asiatici, Istituto universitario orientale, Series Minor XXXIII.

Fratkin, E. 1997. "Pastoralism: governance and development issues". Annual Review of Anthropology, 26:(235-26I).

Gautier, D., Ankogui-Mpok, G.-F., Réounodji, F., Njoya, N., Seignobos, C. 2005. "Agriculteurs et éleveurs des savanes d'Afrique centrale : de la coexistence à l'intégration territori- 
ale". L'espace géographique, 3(34):223-236.

Graefen, C., Kirsch-Jung, K.-P., Banzhaf, M. (2005). "Les impacts socioéconomiques de la gestion décentralisée des ressources naturelles. La contribution des conventions locales à la lutte contre la pauvreté". Rapport du Projet Pilote Droit Foncier et Systèmes fonciers. Réseau Sectoriel Développement Rural Afrique. Groupe de Travail Gouvernance des Ressources Naturelles. Deutsche Gesellschaft für Technische Zusammenarbeit (GTZ) GmbH, Eschborn, Allemagne.

Gonné B., Sougnabé P. et Ngana F. 2010. "Le champ et le bœuf en savane d'Afrique centrale, complémentarité, antagonisme ou intégration ?" pp. 56-69. In Seiny-Boukar, L et Boumard P. (Eds), 20I0. Actes du colloque Savanes africaines en développement : innover pour durer, 20-23 avril 2009, Garoua, Cameroun. Prasac, N'Djaména, Tchad ; Cirad, Montpellier, France, cédérom.

Hagberg, S. 200I. "À l'ombre du conflit violent. Règlement et gestion des conflits entre agriculteurs karaboro et agro-pasteurs peul au Burkina Faso". Cahiers d'études africaines, I6I:(45-72)

Hussein, K., Sumberg, J and Seddon, D. 2000. "Increasing violent conflict between herders and farmers in Africa: claims and evidence". Development policy review, 17:(397-4 I8).

Kossoumna Liba'a, N. 2008. De la mobilité à la sédentarisation : gestion des ressources naturelles et des territoires par les éleveurs Mbororo au Nord du Cameroun. Thèse de Doctorat en géographie et aménage- ment de l'espace, Université PaulValéry Montpellier III, France, 329 p.

Moritz, M. 2006. "Changing contexts and dynamics of herder-farmer conflicts across west Africa". Revue canadienne des études africaines, 40(I): I-40.

Moritz, M. 2006. "The Politics of Permanent Conflict: Herder-Farmer Conflicts in the Far North of Cameroon". Canadian Journal of African Studies, 40(I): 101-126.

Moritz, M. Scholte, P. et Kari, S. 2002. "The Demise of the Nomadic Contract: Arrangements and Rangelands under pressure in the Far North of Cameroon". Nomadic Peoples, (6): I 27- I 46.

Ouattara, S. 1997. "Conflits agriculteurs-éleveurs : l'Etat coupable". Syfia International, Burkina Faso du 01/I I/I997.

Seydou Badian, K. 200I. Sous l'orage. Suivi de la mort de Chaka. Présence Africaine. 253 p.

Sougnabe Pabame, S. 2003. "Conflits agriculteurs-éleveurs en zone soudanienne au Tchad : Une étude comparée de deux régions : MoyenChari et Mayo-Kebbi" pp. 65-82. In Jamin, J.Y., Seiny Boukar, L. et Floret, C. (Eds), 2003. Savanes africaines : des espaces en mutation, des acteurs face à de nouveaux défis. Actes du colloque, mai 2002, Garoua, Cameroun. PRASAC, N'Djamena, TchadCIRAD, Montpellier, France.

Teyssier, A., Hamadou, O. et Seignobos, C. 2003. "Expériences de médiation foncière dans le Nord-Cameroun". Réforme agraire 2003/I, FAO, Département du développement économique et social, Rome. Tonah, S. 2006. "Farmer-Herder Con- 
flict in Volta region of Ghana". Journal of social sciences, 2(I): 6-10 Torre, A., Melot, R., Bossuet, L., Cadoret, A., Caron, A., Darly, S., Jeanneaux, P., Kirat, T. et Vu Pham, H. 2010. "Comment évaluer et mesurer la conflictualité liée aux usages de l'espace ? Eléments de méthode et de repérage". VertigO -
La revue en sciences de l'environnement, IO(I) : avril 20I0. URL : http:/ /vertigo.revues.org/9590.

Van den Brink, R., Bromley, W D. and Chavas, J-P. 1995. "The economics of Cain and Abel: agro-pastoral property rights in the Sahel". Journal of Development Studies 3 I (3):373-399. 